\title{
Terahertz time-domain measurements by electro-optic crystals with various symmetries
}

\author{
I.E. Ilyakov ${ }^{1}$, G.Kh. Kitaeva ${ }^{2}$, B.V. Shishkin ${ }^{1}$, and R.A. Akhmedzhanov ${ }^{1}$ \\ ${ }^{1}$ Institute of Applied Physics RAS, Nizhny Novgorod 603950, Russia, e-mail: igor ilyakov@mail.ru \\ ${ }^{2}$ M.V. Lomonosov Moscow State University, Faculty of Physics, Moscow 119991, Russia
}

Terahertz wave radiation (TWR) is attractive due to its applications in spectroscopy, communication technologies, biology, medical science and other fields. One of the popular ways of TWR time-domain measurements is based on its nonlinear-wave interaction with femtosecond laser pulses in electro-optic (EO) crystals [1]. This technique utilizes ellipticity changes of the laser pulse polarization (the Pockels effect) and is widely used because of its simplicity, high sensitivity and broad operating band of EO crystals. In the report, we present our recent theoretical and experimental study [2-6] of alternative $\mathrm{EO}$ detection techniques and compare them with the standard one in terms of their sensitivity and compatibility with different crystals.

When linearly polarized laser pulse propagates in isotropic crystal (for example $\mathrm{ZnTe}$ or GaP) then in the absence of terahertz electric field its polarization doesn't change. However, in the presence of electric field a refractive index tensor of the crystal is changed and the crystal becomes anisotropic. As a result, orthogonal polarization components of the laser pulse transmit through the matter with different optical densities and the laser pulse polarization becomes elliptic. This polarization ellipticity is measured by an appropriate optical scheme (figure 1,a) and terahertz electric field overlapped with the laser pulse is determined. This technique can be used with TWR generated by sources directed by femtosecond laser pulses [1] and from external sources (not synchronized with the laser system) emitting both continuous [7] and pulsed radiation [8]. In spite of high sensitivity of the technique, we have shown that sensitivity of EO measurements can be increased. The sensitivity improvement can be achieved by use of laser pulses with an edge-cut spectrum [4-6]. A new experimental scheme is presented in figure 1, $b$. An edge filter is positioned before the $\mathrm{EO}$ crystal to produce a high gradient of the spectral energy density of the laser pulse and the TWR interacted with the laser pulse that had previously passed through an edge filter. It was shown theoretically [5] that changes of polarization ellipticity at the frequencies corresponding to the edge-cut of the laser pulse spectrum could be much higher than those could when laser pulse with Gaussian spectrum is used. To utilize the ellipticity changes at the frequencies corresponding to the edge-cut of the laser pulse spectrum, the laser pulse after the EO crystal can be decomposed in the frequency spectrum by a diffraction grating or can be passed through another edge filter transmitting only the optical waves with frequencies corresponding to the slope of the first filter [5, 6] (figure 1, $b$ ). The ellipticity changes are measured using an appropriate optical scheme. In works $[5,6]$ the scheme depicted in figure $1, b$ was implemented with $\mathrm{GaP}$ crystal. This crystal has no natural birefringence and the new tech- nique demonstrated a much stronger response on the same TWR than the standard one (figure 2,a). The method based on ellipticity changes of the laser pulse with an edge-cut spectrum was also successfully used to improve the frequency band of the near-field microscopy technique [9].

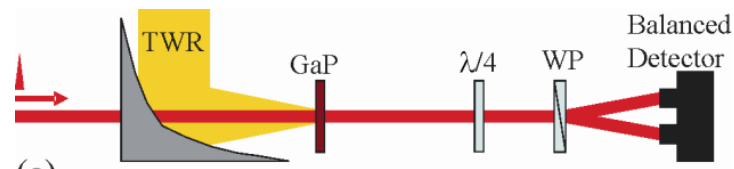

(a)

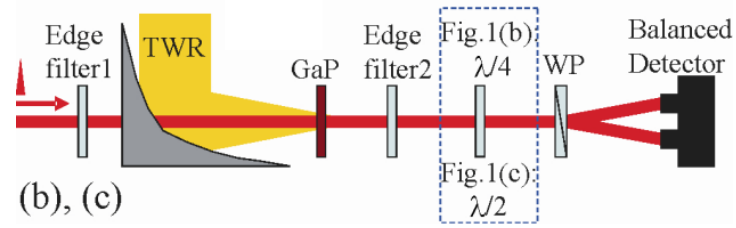

Fig. 1. Schematic of the experimental setup: (a) standard detection scheme; (b), (c) variants of detection schemes with edge filters
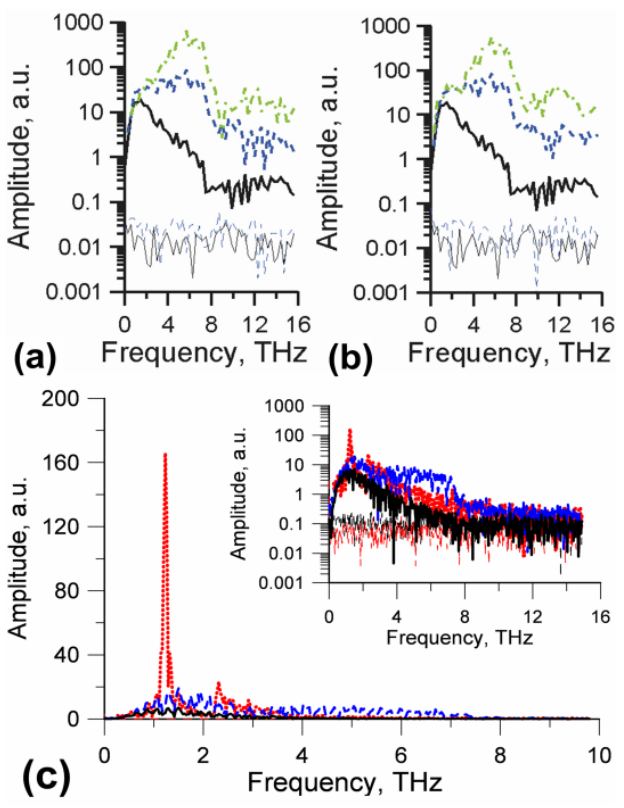

Fig. 2. (a)/(b) Fourier amplitude spectra of time dependences measured by standard measurement scheme (black-solid line) and by the schemes depicted in Figure 1(b)/1(c) with different edge filter 1 orientations (blue-dashed line and green-dasheddot line). (c) Fourier amplitude spectra (inset - a logarithmic scale) of time dependences measured by standard measurement scheme depicted in Figure 1(a) (black-solid line), by the scheme depicted in Figures 1(c) (blue-dashed line) and its analog with PPLN (red-dotted line)

Further progress in sensitivity, frequency range, compactness and cost-efficiency of the EO detection technique can be achieved by application of EO medium 
with higher nonlinearity, having new frequencies of optical-TWR synchronism and being applicable with compact low-cost fiber lasers. A number of well-known organic crystals (DSTMS, DAST and OH1) and PPLN crystals previously successfully used for TWR generation are suitable for making progress in these directions. However, these crystals have high natural birefringence and the use of them with ellipsometric detection technique (figures $1, a$ and $1, b)$ is hindered by decoherence of the laser polarization components [10]. Meanwhile, in the last years sensitive schemes for EO detection based on laser pulse energy changes were suggested [2-6]. We demonstrated both experimentally and theoretically that when the laser pulse interacts with TWR in EO crystal not only its polarization ellipticity but also its energy could be changed $[2,3]$. These changes depend on the amplitude and phase of the TWR overlapped with the laser pulse and can be used for terahertz time-domain measurements [2, 3]. The method based on energy changes can separately measure the energies of the orthogonal polarization components of the laser pulse and, in contrast to techniques based on ellipticity changes, does not require maintaining coherence between the polarization components. Thus, detection schemes based on this method can potentially be applied to birefringent crystals that are promising for further improvement of the sensitivity of EO time-domain measurements. In our research we applied this technique both with isotropic crystals and with birefringent ones [26]. The simplest scheme is based on the total energy changes of the laser pulse. We experimentally demonstrated that it is applicable to terahertz time-domain measurements with the use of birefringent and isotropic EO crystals [2, 3], but for the same TWR electric field and the same isotropic EO crystal ( $\mathrm{GaP}$ or $\mathrm{ZnTe})$ the magnitude of the registered signal was lower compared to the standard detection technique [3]. To improve the sensitivity of the "energy based" method the laser pulses with the edge-cut spectrum were used. As we have shown theoretically $[4,5]$ the relative changes of spectral energy density at the frequencies corresponding to the edge-cut of the laser pulse spectrum can be very high also as changes of polarization ellipticity. To utilize them in experiment the laser pulse after the EO crystal can be decomposed in the frequency spectrum by a diffraction grating [4] or can be passed through another edge filter [5] (figure 1,c) in the same way as in the case of ellipticity changes (figure $1, b$ ). The experimental results obtained with the detection set-up depicted in figure 1, $c$ shows much better response for high TWR frequencies comparing with the standard technique figure $2, b$ ). To approbate the technique with birefringent crystal we used EO crystal - periodically poled lithium niobate (PPLN) and compared its sensitivity with sensitivities of the detection techniques utilizing a GaP crystal [6]. PPLN crystals demonstrate high optical-TWR conversion efficiency for narrow-band generation at various terahertz frequencies and are applicable to achieve quasi-phase-matching between TWR and the laser pulse radiation for different carrier wavelengths. The experimental results show that the use of PPLN for terahertz time-domain measurements further improves the sensitivity of the detection setup at quasi-phase-matching frequencies of about $1.245 \mathrm{THz}$ (figure 2,c) [6]. The proposed technique provides a convenient and effective way of using birefringent crystals in EO time-domain measurements. The use of some other birefringent materials for terahertz time-domain measurements is very interesting in terms of improving the characteristics of the detection technique.

The presented EO detection techniques demonstrate higher sensitivity than the standard one and applicability with greater variety of crystals. The sensitivity of the proposed techniques depends on the parameters of the edge filters and of the optical detectors and their optimization along with the use of other nonlinear materials can lead to further improvement of the sensitivity of EO timedomain measurements.

This work was supported by Russian Foundation for Basic Research (RFBR) Grant Nos. (16-02-01078, 16-0200258, 16-32-00717).

\section{References}

1. Wu, $Q$. and Zhang, $X$.-C. Free-space electro-optic sampling of terahertz beams // Appl. Phys. Lett. 1995. V. 67. P. 3523-3525.

2. Kitaeva, G.Kh., Kovalev, S.P., Naumova, I.I., Akhmedzhanov, R.A., Ilyakov, I.E., Shishkin, B.V., and Suvorov E.V. Quasi-phase-matched probe-energy electro-optic sampling as a method of narrowband terahertz detection // Appl. Phys. Lett. 2010. V. 96. P. 071106.

3. Ilyakov, I.E., Kitaeva, G.Kh., Shishkin, B.V., and Akhmedzhanov, R.A. Laser pulse amplitude changes induced by terahertz waves under linear electro-optic effect // Appl. Phys. Lett. 2014. V. 104. P. 151107.

4. Ilyakov, I.E., Kitaeva, G.Kh., Shishkin, B.V., and Akhmedzhanov, R.A. Terahertz wave electro-optic measurements with optical spectral filtering // Appl. Phys. Lett. 2015. V. 106. P. 121101.

5. Ilyakov, I.E., Kitaeva, G.Kh., Shishkin, B.V., and Akhmedzhanov, R.A. Terahertz time-domain electro-optic measurements by femtosecond laser pulses with an edge-cut spectrum // Opt. Lett. 2016. V. 41. P. 2998-3001.

6. Ilyakov, I.E., Kitaeva, G.Kh., Shishkin, B.V., and Akhmedzhanov, R.A. Electro-optic sampling of terahertz waves by laser pulses with an edge-cut spectrum in birefringent crystal // Opt. Lett. 2017. V. 42, No. 9. P. 1704-1707.

7. Gaal, P., Raschke, M. B., Reimann, K. and Woerner, M. Measuring optical frequencies in the $0-40 \mathrm{THz}$ range with nonsynchronized electro-optic sampling // Nat. Photon. 2007. V. 1. P. 577-580.

8. Barbieri, S., Ravaro, M., Gellie, P., Santarelli, G., Manquest, C., Sirtori, C., Khanna, S. P., Linfield, E. H. and Davies, $A$. $G$. Coherent sampling of active mode-locked terahertz quantum cascade lasers and frequency synthesis // Nat. Photon. 2011. V. 5. P. 306-313.

9. Blanchard, F., Tanaka, K. Improving time and space resolution in electro-optic sampling for near-field terahertz imaging // Opt. Lett. 2016. V. 41. P. 4645-4648.

10. Han, P.Y., Tani, M., Pan, F., and Zhang, X.-C. Use of the organic crystal DAST for terahertz beam applications // Opt. Lett. 2000. V. 25. P. 675-677. 